\title{
"Breakfast like a king, lunch like a prince, and dinner like a pauper": how do European children and adolescents eat?
}

\author{
Leonie Helen Bogl ${ }^{1,2}$, Timm Intemann ${ }^{1,3}$, Monica Hunsberger ${ }^{4}$, Alfonso Siani ${ }^{5}$, \\ Stefaan De Henauw ${ }^{6}$, Dénes Molnár ${ }^{7}$, Luis Moreno ${ }^{8}$, Michael Tornaritis ${ }^{9}$, Toomas Veidebaum ${ }^{10}$, \\ Wolfgang Ahrens ${ }^{1,3}$ and Antje Hebestreit ${ }^{1}$ \\ ${ }^{1}$ Leibniz Institute for Prevention Research and Epidemiology - BIPS, Bremen, Germany, \\ ${ }^{2}$ Department of Epidemiology, Center for Public Health, Medical University of Vienna, Vienna, Austria, \\ ${ }^{3}$ Institute of Statistics, Faculty of Mathematics and Computer Science, University of Bremen, Bremen, Germany, \\ ${ }^{4}$ Department of Public Health and Community Medicine, University of Gothenburg, Gothenburg, Sweden, \\ ${ }^{5}$ Institute of Food Sciences, National Research Council, Avellino, Italy, \\ ${ }^{6}$ Department of Public Health, Ghent University, Ghent, Belgium, \\ ${ }^{7}$ Department of Pediatrics, University of Pécs, Pécs, Hungary, \\ ${ }^{8}$ GENUD (Growth, Exercise, Nutrition and Development) Research Group, Faculty of Health Sciences, University of \\ Zaragoza, Zaragoza, Spain, \\ ${ }^{9}$ Research and Education Institute of Child Health, Strovolos and \\ ${ }^{10}$ Department of Chronic Diseases, National Institute for Health Development, Tallinn, Estonia
}

\begin{abstract}
Chrono-nutrition is an emerging field of research that focuses on the interplay between nutrition, circadian rhythms and metabolism. Most nutritional guidelines recommend regular and frequent meals for children and adolescents throughout the day. However, preliminary research, mostly in animals suggests that eating at the "wrong" time of the day and longer eating windows (and concomitant shorter fasting periods) may relate to metabolic health. This study aimed to identify meal-timing patterns of European children and adolescents in eight European countries.

We examined 1225 children and adolescents (mean age: 11.8 years, 50\% boys, and 26\% overweight/obese) from Belgium, Cyprus, Estonia, Germany, Hungary, Italy, Spain and Sweden enrolled in the European I.Family study (2013/2014). Information on energy intake and meal-timing patterns was derived from multiple web-based 24-hour dietary recalls (proxy-assisted for children $<12$ years). We used the National Cancer Institute (NCI) method to estimate individual usual intakes. Subsequently, we applied the k-means algorithm to identify clusters for meal-timing patterns. Five variables were selected for the cluster analysis: 1) Duration between first and last meal of the day (eating window in hours), 2) Proportion of daily energy intake before 11am, 3) Proportion of daily energy intake after 5pm, 4) Number of meals per day, and 5) Pre-sleep fasting time.

Three clusters, labelled "late and time-restricted", "late and long" and "early and frequent" meal-timing patterns were identified. Similar clusters were derived when excluding energy misreporters according to Goldberg cut-offs. Children in the "early and frequent" pattern were younger, had a lower body mass index (BMI) z-score, and a longer sleep duration than children in the other two mealtiming patterns. A higher proportion of plausible energy reporters were classified into the "early and frequent" pattern than into the other two patterns. The proportion of children from Italy, Cyprus and Spain was highest in the "late and long" pattern, while the proportion of children from Belgium, Sweden, Germany and Hungary was highest in the "early and frequent" patterns, and the proportion of children from Estonia was highest in the "late and time-restricted" pattern.

In conclusion, this study identified three different meal-timing patterns in children that varied by age, BMI z-score, country, misreporting status, and sleep duration. Investigations on the associations between meal timing patterns and metabolic health in this study sample are currently ongoing.
\end{abstract}

\section{Conflict of Interest}

There is no conflict of interest 